\title{
An Overview on Dynamics and Controls Modelling of Hypersonic Vehicles
}

\author{
Michael A. Bolender \\ Control Design and Analysis Branch \\ Air Force Research Laboratory \\ Wright-Patterson AFB, OH 45433 \\ Michael.Bolender@us.af.mil
}

\begin{abstract}
In order to appropriately design control laws for hypersonic vehicles, it is paramount to understand how the flight dynamics are impacted by the interactions between the aerothermodynamics, propulsion system, structural dynamics, and control system. To this end, there has been a significant investment into the modelling of these sub-systems and their integration into a comprehensive model that can be used to the characterize the flight dynamics of scramjet-powered hypersonic aircraft and still remain amenable to control law design and analysis. In this paper, the development of a comprehensive model of the longitudinal dynamics of generic hypersonic vehicle with an outward-turning, two-dimensional inlet is described. The sub-system models, for the most part, are simple models derived from first-principles and are intended to capture the interactions between the different sub-systems to provide a representative vehicle model. We also will discuss the areas that are important to realizing a hypersonic modelling approach that can take any given vehicle geometry and permit a thorough analysis of its stability and control characteristics and any practical constraints on its operability, the design of a control law, an assessment of it closed-loop performance.
\end{abstract}

\section{INTRODUCTION}

Air-breathing hypersonic aircraft are seen as a possible solution to making access to space routine and affordable. Research into air-breathing hypersonics began during the 1960's and continued through the 1990's with the National Aerospace Plane. In spite of this long period of research, it is widely recognized that the development of a full scale operational vehicle will require major advances in propulsion and materials technology as well as more mature multidisciplinary modelling and design tools. After the cancellation of the National Aerospace Plane in the early 1990's, research into scramjet-powered flight continued, although on a much smaller scale. The subject of few programs was to demonstrate the feasibility of component technologies needed to achieve sustained hypersonic flight. The most recent example has been NASA's successful flight tests of the scramjet powered X-43A, a sub-scale technology demonstrator, that flew in 2004 and 2005. Later in 2009, it is expected that the X-51 Scramjet Engine Demonstrator will make its maiden flight.

The design of air-breathing hypersonic vehicles typically have a tightly integrated airframe and scramjet propulsion system. A "traditional" scramjet-powered vehicle design has an extended forward fuselage that creates a series of bow shocks that act as a compression system for the scramjet engine. The pressure distribution on this forebody produces lift and a nose-up pitching moment. Downstream of the scramjet engine, the aircraft is shaped such that there is an external expansion of the exhaust gases. The pressure on the aftbody is a function of the pressure and Mach Number of the exhaust gas as it exits the scramjet engine. As this gas expands the pressure applied to the aftbody contributes to the lift of the vehicle, partially offsets the drag, and provides the vehicle with a nose-down pitching moment. With the engine below the center-of-gravity, the thrust produces a nose-up pitching moment that must be balanced by the aerodynamics and control surfaces. The structural modes for this class of vehicle also play an important role. The vibrating, forward fuselage effectively changes the pressure distribution over the forebody of the aircraft since it changes the apparent turn angle of the flow relative to the freestream. The resulting changes in the pressure distribution over the aircraft are realized downstream as perturbations to the inlet boundary conditions and also as lift, drag and pitching moment perturbations.

In the literature, there have been several papers that discuss the challenges associated with the dynamics and control of air-breathing hypersonic vehicles (HSVs) [1], [2]. The first attempt at a comprehensive analytical model of the longitudinal dynamics of an HSV was undertaken by Chavez and Schmidt [3]. Their use of Newtonian Impact Theory provided for a analytical expressions for the pressure distribution on the vehicle. The pressures were dependent upon vehicle Mach number, freestream pressure, angle-of-attack, and the vehicle geometry. These expressions were then manipulated to give analytical expressions for the total aerodynamic forces on the vehicle. The resulting equations were then linearized to give analytical expressions for the stability and control derivatives. Ultimately, requirements and control laws for the Chavez/Schmidt HSV were synthesized. [1], [4].

In this paper, we review one particular effort to develop a model of a scramjet powered hypersonic vehicle that captures the structural, aerothermodynamic, and propulsion system coupling inherent in a scramjet powered vehicle. The focus to date has been solely on a configuration with an outwardturning, 2-dimensional inlet. The approach described herein builds on the model of Chavez and Schmidt [3], with different approaches taken to address the influence of additional couplings that were expected to exist [2] at the time, but whose effects on vehicle stability and control were uncertain. The resulting model of the longitudinal dynamics of an air- 
breathing hypersonic vehicle captures many of the effects of the diverse physical phenomena that present challenges to flight control law designers. The form of the model is suitable for use in configuration trade-studies early in the design process where designers are interested in developing a configuration that is amenable to feedback control.

\section{FIRST-PRINCIPLES MODELLING FOR DYNAMICS AND CONTROL ANALYSIS}

For the past few years, there has been a concerted effort to develop a first-principles based model of a generic airbreathing hypersonic vehicle. The purpose of this effort is to develop a modelling environment that control engineers can use early in the design process to help understand the physical manifestation of the complex interactions between the aerothermodynamics, propulsion system, control system, and structural dynamics that occurs for a given configuration. Such models allow flight control engineers to obtain a fundamental understanding of the effects of these interactions on the open-loop dynamics of the system, and then be able to quickly determine the trade-space to enhance the controllability of the vehicle. For example, the configuration might be modified by the addition of a new control effector or by making a change to the outer mold line that results in improved controllability.

The philosophy of this research effort has been to incrementally add complexity to the model. This approach allows for each refinement to be assessed in terms of its relative impact on the open-loop flight dynamics of the vehicle as compared to the "original" model. As can be expected, the model has undergone a rather significant modification with regard to its fidelity. We still maintain the philosophy that the aerodynamics forces and moments are not stored in look-up tables, but instead are calculated at each time step of the simulation given the actuation of the controls and the current state of the vehicle. For example, in the original vehicle model [5], it was assumed that the airflow over the vehicle was quasi-steady. This assumption allowed us to use oblique-shock theory and Prandtl-Meyer flow to determine the pressure distribution on the vehicle. However, the downfall of this approach was that there was no means by which one could calculate the aerodynamic damping derivatives or the unsteady aerodynamic forces and moments due to the fluid-structure interaction that arises when the vehicle vibrates. To remedy this, linear piston theory has been utilized to capture the unsteady components of the flow field [6], [7]. Additionally, because the original model assumed inviscid flow, an analytical skin friction model using Eckert's reference temperature method has been incorporated into the model [8] to give more realistic drag estimates.

Additionally, changes have been made to the aeroelastic model to improve the estimation of the mode shapes and frequencies of the structural dynamics [9]. The initial structural model was limited because it could only approximate the first mode shape of the structure, and it was difficult to capture mass and temperature effects on the mode shapes of the vehicle. By utilizing the Assumed Modes Method [9], we are now able to calculate any desired number of frequencies and mode shapes for the vehicle. One benefit of the Assumed Modes Method is that we know longer have the inertial coupling that was present in the original model, which was a direct result of our choice of a model for the structural dynamics. Additionally, we can now model the evolution of the frequencies and mode shapes throughout the trajectory as the mass of the vehicle changes and the temperature of the structure increases due to aerodynamic heating. The capability provided further motivation to develop an unsteady heat transfer model [10] that would allow us to estimate the aerodynamic heating on the vehicle in order to calculate the temperature of the underlying structure. The output of the unsteady heat transfer model is an average temperature distribution that subsequently becomes an input to the assumed modes code to accommodate the change in material properties due to temperature.

The purpose of this paper is to provide an engineering analysis of the vehicle's flight dynamics with the above improvements incorporated into the model. The impact of the increased model fidelity is used to determine the appropriate level of modelling needed to obtain a reasonable approximation to the flight dynamics of air-breathing hypersonic vehicles.

\section{A. Model Description}

The stability axis longitudinal equations-of-motion for a flexible aircraft flying over a spherical earth are given by [11]

$$
\begin{aligned}
\dot{V}_{t} & =(T \cos \alpha-D) / m-g \sin \gamma \\
\dot{\alpha} & =-(T \sin \alpha+L) /\left(m V_{t}\right)+\left(g / V_{t}-V_{t} / r\right) \cos \gamma+Q \\
\dot{Q} & =\mathscr{M} / I_{y y} \\
\dot{\Theta} & =Q \\
\dot{h} & =V_{t} \sin \gamma \\
\ddot{\eta}_{i} & =-2 \zeta \omega_{i} \dot{\eta}_{i}-\omega_{i}^{2} \eta_{i}+N_{i}, i=1,2, \ldots, n
\end{aligned}
$$

where $V_{t}$ is the true airspeed, $T$ is the thrust, $D$ and $L$ are the drag and lift respectively, $\alpha$ is the angle-of-attack of the vehicle, $\gamma=\Theta-\alpha$ is the flight path angle, $\mathscr{M}$ is the pitching moment, $Q$ is the pitch rate, $h$ is the altitude, $r=R_{E}+h$ is the radius of the vehicle measured with respect to the earth's center, $\eta_{i}$ is the $i^{t h}$ modal coordinate of the flexible dynamics, $\zeta$ and $\omega_{i}$ are the damping ratio and the natural frequency respectively, and $N_{i}$ is the $i^{t h}$ generalized force, where

$$
N_{i}=\int_{B} p(x, t) \Phi_{i}(x) d x+\sum_{j} F_{j}(t) \Phi_{i}\left(x_{j}\right)
$$

and $p(x, t)$ is the pressure distribution over the vehicle, $\Phi_{i}(x)$ is the $i^{t h}$ mode shape, $F_{j}(t)$ is the $j^{t h}$ point load acting at a point $x_{j}$ on the vehicle. The acceleration due to gravity is given by the inverse-square law

$$
g=g_{0}\left(\frac{R_{E}}{R_{E}+h}\right)^{2}
$$


where $g_{0}$ is the acceleration due to gravity at sea level and $R_{E}$ is the radius of the earth.

The coupling between the structural dynamics and rigidbody dynamics for this particular dynamical model occurs through the aerodynamic forces and moments. In particular, if we consider the pressure acting on any surface in Figure 1, we can see that it has its usual dependency upon the Mach number, altitude, and angle-of-attack. This is evident through the equation for the pressure behind an oblique shock (assuming a perfect gas)

$$
\frac{p_{2}}{p_{1}}=\frac{7 M_{1}^{2} \sin ^{2} \theta_{s}-1}{6}
$$

where $p_{2}$ is the pressure behind the shock, $p_{1}$ is the pressure ahead of the shock, $M_{1}$ is the Mach number of the flow upstream of the shock, and $\theta_{s}$ is the shock angle. The shock angle is a function of Mach number and the flow turn angle, and is found by solving the following equation [12] for $\theta_{s}$ :

$$
\begin{aligned}
\sin ^{6} \theta_{s} & +b \sin ^{4} \theta_{s}+c \sin ^{2} \theta_{s}+d=0 \\
b & =-\frac{M_{1}^{2}+2}{M_{1}^{2}}-(7 / 5) \sin ^{2} \delta \\
c & =\frac{2 M_{1}^{2}+1}{M_{1}^{4}}+\left(\frac{72}{50}+\frac{2 / 5}{M_{1}^{2}}\right) \sin ^{2} \delta \\
d & =-\frac{\cos ^{2} \delta}{M_{1}^{4}}
\end{aligned}
$$

where $\delta$ is the angle through which the flow is compressed. Likewise, for an expansion over a surface, the pressure acting on the surface can be found from the thermodynamic relation for an isentropic flow from

$$
\frac{p_{2}}{p_{1}}=\left[\frac{1+(1 / 5) M_{1}^{2}}{1+(1 / 5) M_{2}^{2}}\right]^{(7 / 2)}
$$

For an expansion, $M_{2}$ is determined directly from the Prandtl-Meyer Equation [12].

$$
f\left(M_{2}\right)=\sqrt{6} \tan ^{-1} \sqrt{6\left(M_{2}^{2}-1\right)}-\tan ^{-1} \sqrt{M_{2}^{2}-1}-\nu_{2}
$$

where $\nu_{2}$ is the total expansion angle of the flow. Because both expressions for the pressures given above are ultimately functions of the angle of the local surface with respect to the freestream, there is an implied dependency on the angle-ofattack. However, the local angle-of-attack will be perturbed by the deflection of the vehicle structure. Therefore, the local flow conditions, and thus the aerodynamic forces, are also dependent upon the magnitude of this effective change in angle-of-attack.

1) Piston Theory: The aerodynamic model uses Linear Piston Theory to compute the unsteady pressure distribution on the vehicle that results from both flow-structure interaction and the unsteady, rigid body motion of the vehicle. Piston Theory has been used extensively by aeroelasticians to determine the pressure distributions on a flexible airfoil when the Mach number is sufficiently high. Lighthill [13] first discussed the application of piston theory on oscillating

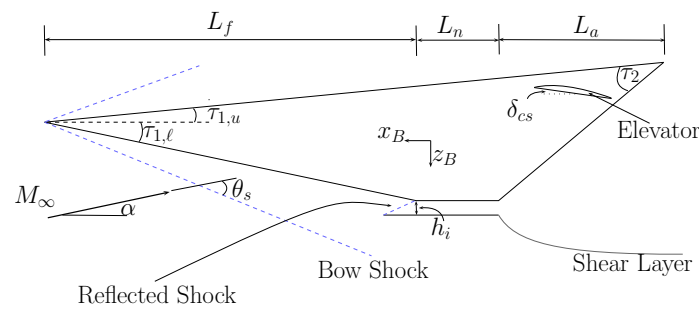

Fig. 1. Hypersonic Air-breathing Vehicle Geometry

airfoils some 50 years ago. Ashley and Zartarian [14] discuss piston theory while providing a number of examples of the application of piston theory to specific problems. More recently, Tarpley [15] discussed the computation of stability derivatives for a caret-wing waverider using Piston Theory, which requires the analysis of unsteady flow over the vehicle [15]. Piston theory allows the inclusion of unsteady aerodynamic effects in the model and a closed form solution can be found for these unsteady effects.

Linear Piston Theory states that the pressure acting on the face of a piston moving in a perfect gas when the Mach number is greater than unity is [14]:

$$
\frac{P}{P_{i}}=\left(1+\frac{1}{5} \frac{V_{n, i}}{a_{i}}\right)^{7}
$$

where $P$ is the pressure on the piston face, $P_{i}$ is the local static pressure (i.e., behind the shock in the case of supersonic flow and assumed to be constant), $a_{i}$ is the local speed of sound, and $V_{n, i}$ is the velocity of the surface normal to the steady flow. Taking the binomial series of Equation 13 to first order gives

$$
\frac{P}{P_{i}}=1+\frac{7}{5} \frac{v_{n, i}}{a_{i}}
$$

Multiplying through by $P_{i}$, using the perfect gas law, and the definition for speed of sound gives the basic result from first-order linear piston theory:

$$
P=P_{i}+\rho_{i} a_{i} v_{n, i}
$$

where $\rho_{i}$ is the local density of the fluid. The normal velocity, $v_{n, i}$ is now a perturbation to the velocity $V_{n, i}$, and arises due to either the flexing of the aircraft, the aircraft's rotational motion, or a change in angle-of-attack from $V_{n, i}$.

The infinitesimal force acting on the face of the piston is

$$
d \boldsymbol{F}_{i}=-(P d A) \boldsymbol{n}_{i}
$$

In Equation 16, $\boldsymbol{n}$ is the outward pointing normal unit vector to the surface, $d A$ is the infinitesimal surface area, and $d \boldsymbol{F}_{i}$ is the incremental force. Substituting Equation 15 into Equation 16 gives

$$
d \boldsymbol{F}_{i}=\left[-\left(P_{i}+\rho_{i} a_{i} v_{n, i}\right) d A\right] \boldsymbol{n}_{i}
$$

However, since $v_{n, i}$ is by definition the perturbation velocity normal to the surface, we can write $v_{n, i}=\boldsymbol{v} \cdot \boldsymbol{n}_{i}$ where $\boldsymbol{v}$ 
is the perturbation velocity vector of the vehicle. Thus, the infinitesimal force becomes

$$
\left.d \boldsymbol{F}=-\left\{\left[P_{i}+\rho_{i} a_{i}\left(\boldsymbol{v} \cdot \boldsymbol{n}_{i}\right)\right] d A\right]\right\} \boldsymbol{n}_{i}
$$

Equation 18 is then integrated over each surface that defines the vehicle outer mold line to give the total force acting on the vehicle. From Equation 18 it should be noted that when $\int P_{i} d A \boldsymbol{n}_{i}$ is evaluated over the vehicle, this contribution will give exactly the quasi-static pressure distribution that is calculated in Reference [5]. The unsteady contribution comes from the normal component of the velocity for a given surface, $\boldsymbol{v}_{n} \cdot \boldsymbol{n}$. It is the unsteady contribution that will give rise to the damping derivatives, $M_{q}$, for example, and for lack of a better term, the structural damping derivatives, $M_{\dot{\eta}}$, etc. A thorough treatise on modelling the unsteady aerodynamics with piston theory for our generic vehicle is given in Oppenheimer [7].

2) Viscous Drag Modelling: The integration of the pressure distribution on the vehicle will only give us the pressure drag. However, the primary source of drag is due to viscous friction of the fluid moving relative to the vehicle. The skin friction drag is proportional to the shear stress of the fluid acting at the vehicle. The local skin friction coefficient is defined as

$$
c_{f}=\frac{\tau_{w}}{(1 / 2) \rho_{\infty} V_{\infty}^{2}}
$$

where $\tau_{w}$ is the shear stress at the wall and $\rho_{\infty}$ and $V_{\infty}$ are the freestream density and velocity respectively. The local shear stress at the wall can be expressed as [16]

$$
\tau_{w}=\left.\mu \frac{\partial u}{\partial y}\right|_{w}
$$

where $\mu$ us the viscosity of the fluid, and $\partial u / \partial y$ is the velocity gradient through the boundary layer.

To calculate the drag, we will calculate the skin friction drag for each wetted surface on the vehicle. For our purposes we will assume that the boundary layer will be turbulent over the entire vehicle. This assumption is made to impart conservativeness in our skin friction drag calculation. Furthermore boundary layer transition is not well understood, even for flow over a flat plate, and therefore it is difficult to predict the transition from laminar to turbulent flow. We will employ a method attributed to Eckert that utilizes the concept of a reference temperature that takes into account the wall temperature.

The skin friction coefficient for turbulent, supersonic flow over a flat plate is given by [16]:

$$
c_{f}=\frac{0.0592}{\left(R e^{*}\right)^{1 / 5}}
$$

where $R e^{*}$ is the Reynolds number $(\rho V L / \mu)$ evaluated at a reference temperature. The reference temperature, $T^{*}$ is determined by

$$
T^{*}=T_{e}\left[1+M_{e}^{2}+0.58\left(\frac{T_{w}}{T_{e}}-1\right)\right]
$$

In Equation 22, $M_{e}$ is the Mach number at the edge the boundary layer (i.e., downstream of the shock or expansion),
$T_{e}$ is the temperature at the edge of the boundary layer, $T_{w}$ is the wall temperature. From Reference [10],a steady-state wall temperature of $2500^{\circ} \mathrm{R}$ was assumed Once the reference temperature $T^{*}$ is known, the density at the reference temperature is found from the perfect gas law using the static pressure of the fluid

$$
\rho^{*}=\frac{p}{R T^{*}}
$$

The viscosity at the reference temperature, $\mu^{*}$ is then calculated using Sutherland's Formula [12]

$$
\mu^{*}=2.27 \times 10^{-8} \frac{\left(T^{*}\right)^{3 / 2}}{T^{*}+198.6}
$$

which is valid to approximately $3400^{\circ} \mathrm{R}$.

Once the reference Reynolds number has been calculated, the local skin friction coefficient can be calculated at any point along the vehicle using Equation 21. To determine the skin friction drag, we solve Equation 19 for $\tau_{w}$ and integrate over each surface of the vehicle and sum the contributions. Further development of the viscous forces is given in Reference [8].

\section{B. Propulsion System Modelling}

Most recently, there has been substantial work to improve the propulsion system modelling. Originally, a simple, firstprinciples model identical to that in Chavez and Schmidt [3] was used. This model assumed an isentropic internal nozzle and diffuser, and the combustion process was modelled as Rayleigh flow (flow in a constant area duct with heat addition). The control input for the engine was defined to be the equivalence ratio. The equivalence ratio can be directly related to the change in total temperature that results from the combustion process given an assumed combustion efficiency and fuel. Additionally, we can determine the mass flow that is captured by the propulsion system from the flight condition (Mach and angle-of-attack) in order to compute the thrust. This avoids having to make a priori assumptions regarding the fuel schedule and the parameters used for scheduling. While this propulsion model gives a thrust variation with equivalence ratio that is representative of what is expected in a scramjet engine, it lacks fidelity and therefore does not capture several important physical processes that occur in a scramjet engine that are known to have a significant impact on its operability. An improved scramjet model will provide more realistic bounds on engine operability and subsequently impose more realistic and stringent constraints on the vehicle dynamics in order to prevent, for example, combustion flameout during a dynamic maneuver of the vehicle.

The new propulsion model and its effect on the vehicle dynamics is outlined in Torrez and Driscoll [17], and extends previous models found in the literature. O'Brien, Starkey, and Lewis [18], Starkey [19], Birzer and Doolan [20], and Tetlow and Doolan [21] have all constructed propulsion system models of varying fidelity to support hypersonic vehicle design studies. The model defined in Reference [17] extends those above by including a pre-combustor shock train, fuel mixing, and finite-rate chemistry. Currently this is still a 


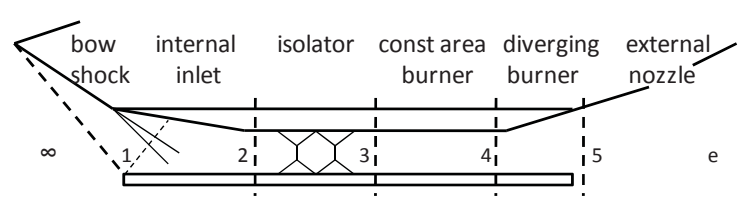

Fig. 2. Schematic of Dual-Mode Scramjet Engine

quasi-steady model as no unsteady flow terms are included in the model.

The propulsion model now consists of a set of ordinary differential equations that govern the heat addition to a variable area duct, which are derived by Shapiro [22]. The mixing model that is incorporated is based on recent experiments of jets mixing in supersonic cross flows. The 18 step finite-rate chemistry mechanism of Zambon and Chelliah [23] was added to account for the use of either ethylene, hydrogen, or methane fuel, and has been carefully validated in Reference [23] for high temperature reactants that are in the auto-ignition regime. Gas dissociation and variable heat capacity are included to model the stagnation temperature loss that occurs due to dissociation. Some of this loss can be recovered in the nozzle provided that there is sufficient time for species recombination. Wall heat transfer and skin friction effects also are included in the model.

The engine flow path model is shown in Figure 2. The geometry is that of a constant area combustor that is $9.84 \mathrm{ft}$ long, followed by a nozzle that is $32.8 \mathrm{ft}$ long. The nozzle exit area is 3.1 times the combustor area. Note that the combustor section consists of two parts: a nearly constant area burner (3-4) into which the mass flow rate of fuel is injected, $\dot{m}_{f, 34}$, followed by a diverging area burner (4-5) into which the fuel injected is $\dot{m}_{f, 45}$. For reasons described in Reference [24], during ramjet operation fuel must be injected into both burners, while for scramjet operation fuel is only injected into the constant-area section. Results for this propulsion model are given in Reference [17].

\section{Flexible Aircraft Model}

The assumed modes method is a technique for computing the natural frequencies and mode shapes of a given flexible structure. It is particularly well suited to the analysis of preliminary vehicle designs of the type under study here, as a full finite element model of the structure is neither required nor desired because such models require extensive calculations and therefore are not suitable because they would significantly affect the speed of the dynamic simulation.

The assumed modes method is based on Lagrange's Equations, and expresses the displacement along the structure as an expansion of the form

$$
w(x, t)=\sum_{i=1}^{n} \Phi_{i}(x) w_{i}(t)
$$

where the shape functions $\left\{\Phi_{i}(x), i=1, \ldots, n\right\}$ are the assumed modes. These functions are termed admissible functions, meaning that they are linearly independent, satisfy all of the geometric boundary conditions of the structure, and possess all required derivatives. The shape functions are used, along with estimates of the mass and stiffness distribution to form a "mass matrix" and a "stiffness matrix" respectively. The elements of the mass matrix are

$$
m_{i j}=\int_{0}^{L} \rho A(x) \Phi_{i}(x) \Phi_{j}(x) d x
$$

where $\rho A(x)$ denotes the mass per unit length of the structure. Likewise, the elements of the stiffness matrix are

$$
k_{i j}=\int_{0}^{L} E I(x) \frac{\partial^{2} \Phi_{i}(x)}{\partial x^{2}} \frac{\partial^{2} \Phi_{j}(x)}{\partial x^{2}} d x
$$

where $E I(x)$ is the stiffness per unit length. Note that $m_{i j}=$ $m_{j i}$ and $k_{i j}=k_{j i}$ for all $i$ and $j$, so the matrices $M$ and $K$ are always symmetric.

The natural frequencies and mode shapes of the structure are then obtained from the unforced harmonic dynamics of the system resulting in an eigenvalue problem given by:

$$
\left(\omega^{2} I-M^{-1} K\right) \boldsymbol{w}=\mathbf{0}
$$

The natural frequencies of the structure are thus the square roots of the eigenvalues of $M^{-1} K$. The corresponding mode shapes are given as linear combinations of the assumed modes, with the coefficients of these expansions given by the eigenvectors of $M^{-1} K$. For results and more detail, the reader is referred to Reference [9].

1) Aerothermoelasticity: The inclusion of aerothermal effects on the structural dynamics is ongoing. For this particular model, a representative structure and thermal protection system suitable for a heat transfer analysis was designed, but is omitted here for brevity. The interested reader is referred to References [10] and [25] for more details. For purposes of this model, it will suffice to assume a temperature distribution for the structure for the sole purpose of calculating the vehicle's frequencies and mode shapes at different points along the mission. A more detailed analysis of mass and temperature effects on the linearized aircraft dynamics is given in Reference [25].

2) Tail-Wags-Dog Effect: This work is the subject of on-going research. The motivation being to capture the interaction between the inertial effects of the control surface and the structural dynamics of the vehicle. The tail-wags-dog effect may be significant given that the elevators are typically all-moving surfaces with large chords relative to the length of the vehicle. The tail-wags-dog effect typically manifests itself as a complex-conjugate pair of zeros in the elevatorto-pitch rate transfer function, thus affecting the speed-ofresponse of the system.

\section{FUTURE WORK}

While the modelling approach outlined above captures the relevant interactions between the aerodynamics, propulsion system, structural dynamics, and the control system, it is limited by the fact that only the longitudinal dynamics of the vehicle are captured. Furthermore, the uncertainty in the model has not been quantified, although it is expected to be 
significant. Current research is being conducted to compare the first-principles model to CFD and FEM codes. Also a new class of models is being developed. These models are reduced-order models that are derived from the output of these high fidelity codes. As a research area, there are still significant advances that need to be made in developing physics-based and reduced-order models, especially as they pertain to the aerothermodynamics of the vehicle. The primary difficulty in first-principles modelling comes in being able to compute the flow field about the vehicle accurately and in a manner that is computationally efficient enough to lend itself to control system analysis, flight simulation, and multi-disciplinary design optimization. Some of the subject areas that need to be addressed are as follows (listed in no particular order):

1) Modelling the on-set of flow separation on control effectors

2) Accurate spill-drag prediction

3) Modelling aftbody aerodynamics and engine exhaust flow physics for vehicles with scarfed nozzles

4) Aerodynamic characterization of streamline traced inlets

5) A priori prediction of inlet and engine operability limits

6) Modelling inlet unstart and its effects on vehicle stability and control

7) Full 3D aerodynamic characterization of a given geometry germane to full 6DOF simulation capabilities

8) Development of an integrated analysis and simulation environment

9) Development of accurate first-principles and reducedorder models that do not require CFD \& FEM codes to be run a priori on the vehicle geometry

\section{CONCLUSION}

In this paper, the development of a comprehensive model of the longitudinal dynamics of generic hypersonic vehicle with an outward-turning, two-dimensional inlet was described. The sub-system models, for the most part, are simple models derived from first-principles, and are intended to capture the interactions between the different sub-systems to provide a representative vehicle model. We also discussed the areas that are important to realizing a hypersonic modelling approach that can take any given vehicle geometry and permit a thorough analysis of its stability and control characteristics, the design of a control law, an assessment of it closed-loop performance, and any practical constraints on its operability.

\section{ACKNOWLEDGEMENT}

This paper has been cleared for public release: Case Number 88ABW-2008-0031.

The author would like to acknowledge the contributions of M.W. Oppenheimer and D.B. Doman to this body of work.

\section{REFERENCES}

[1] D. Schmidt, "Dynamics and control of hypersonic aeropropulsive/aeroelastic vehicles."

[2] D. McRuer, "Design and modeling issues for integrated airframe/propulsion control of hypersonic flight vehicles," in Proceedings of the 1992 American Control Conference, Boston, MA, June, 1991, pp. $729-34$

[3] F. Chavez and D. Schmidt, "Analytical aeropropulsive/aeroelastic hypersonic-vehicle model with dynamic analysis," Journal of Guidance, Control, and Dynamics, vol. 17, no. 6, pp. 1308-1319, Nov-Dec 1994.

[4] D. Schmidt and J. Velapoldi, "Flight dynamics and feedback guidance issues for hypersonic airbreathing vehicles," in AIAA 1999-4122.

[5] M. A. Bolender and D. B. Doman, "Nonlinear Longitudinal Dynamical Model of an Air-Breathing Hypersonic Vehicle," Journal of Spacecraft and Rockets, vol. 44, no. 2, pp. 374-387, March/April 2007.

[6] M. W. Oppenheimer and D. B. Doman, "A hypersonic vehicle model developed with piston theory," in AIAA 2006-6637.

[7] M. W. Oppenheimer, D. B. Doman, M. A. Bolender, and T. Skujins, "A flexible hypersonic vehicle model developed with piston theory," in AIAA 2007-6396.

[8] M. W. Oppenheimer and D. B. Doman, "Viscous effects for a hypersonic vehicle model," in In preparation for submittal to the 2008 AIAA Atmospheric Flight Mechanics Conference.

[9] T. Williams, M. A. Bolender, D. B. Doman, and O. Morataya, "An Aerothermal Flexible Mode Analysis of a Hypersonic Vehicle," in AIAA 2006-6647.

[10] M. A. Bolender and D. B. Doman, "Modeling Unsteady Heating Effects on the Structural Dynamics of a Hypersonic Vehicle ," in AIAA 2006-6646.

[11] K. Bilimoria and D. Schmidt, "Integrated Development of the Equations of Motion for Elastic Hypersonic Flight Vehicles ,"Journal of Guidance, Control, and Dynamics, vol. 18, no. 1, pp. 73-81, 1995.

[12] "Equations, tables, and charts for compressible flow," National Advisory Committee for Aeronautics, Ames Aeronautical Laboratory, Moffett Field, CA, Tech. Rep. NACA-1135, 1953.

[13] M. Lighthill, "Oscillating Airfoils at High Mach Number," Journal of the Aeronautical Sciences, vol. 20, no. 6, pp. 402-406, 1953.

[14] H. Ashley and G. Zartarian, "Piston theory - a new aerodynamic tool for the aeroelastician," Journal of the Aeronautical Sciences, vol. 23, no. 12 , p. $11091118,1956$.

[15] C. Tarpley and M. Lewis, "Stability Derivatives for a Hypersonic Caret-Wing Waverider," Journal of Aircraft, vol. 32, no. 4, pp. 795803.

[16] J. D. Anderson, Hypersonic and High Temperature Gas Dynamics. American Institute of Aeronautics and Astronautics, 1989.

[17] S. M. Torrez, N. Scholten, D. Micka, J. F. Driscoll, M. Bolender, D. Doman, and M. W. Oppenheimer, "A scramjet engine model including effects of precombustion shocks and dissociation," AIAA 2008-4619.

[18] T. F. O'Brien, R. P. Starkey, and M. J. Lewis, "Quasi-one-dimensional high-speed engine model with finite-rate chemistry," Journal of Propulsion and Power, vol. Vol. 17, No. 6, pp. pp. 1366-1374, 2001.

[19] R. P. Starkey, "Off-design performance characterization of a variable geometry scramjet," AIAA 2005-3711.

[20] C. Birzer and C. Doolan, "Quasi-one-dimensional modeling of hydrogen fuelled scramjet combustors," AIAA 2007-4314.

[21] M. Tetlow and C. Doolan, "Comparison of hydrogen and hydrocarbonfueled scramjet engines for orbital insertion," Journal of Spacecraft and Rockets, vol. Vol. 44, No. 2, pp. pp. 365-372, 2007.

[22] A. H. Shapiro, Dynamics and Thermodynamics of Compressible Fluid Flow. Ronald Press, NY, 1953.

[23] A. C. Zambon and H. K. Chelliah, "Explicit reduced reaction models for ignition, flame propagation, and extinction of c2h4/ch4/h2 and air systems," Combustion and Flame, vol. Vol. 150, pp. pp. 71-91, 2007.

[24] W. Heiser and P. D., Hypersonic Airbreathing Propulsion. 370 L'Enfant Promenade, SW,Washington D.C., 20024-2518: American Institute of Aeronautics and Astronautics, 1994, pp. 109-143.

[25] A. Culler, T. Williams, and M. Bolender, "Aerothermal Modeling and Analysis of a Hypersonic Vehicle," in AIAA-2007-6395. 